\title{
Implicit Visuomotor Processing for Quick Online Reactions Is Robust against Aging
}

\author{
Koji Kadota ${ }^{1}$ and Hiroaki Gomi ${ }^{1,2}$ \\ ${ }^{1}$ Shimojo Implicit Brain Function Project, Japan Science and Technology Agency, and ${ }^{2}$ NTT Communication Science Laboratories, Nippon \\ Telegraph and Telephone Corporation, Atsugi, Kanagawa 243-0198, Japan
}

It is well established that humans can react more quickly to a visual stimulus in the visual field center than to one in the visual periphery and that the reaction to a stimulus in the visual periphery markedly deteriorates with aging. These tendencies are true in conventional discrimination-reaction tasks. Surprisingly, however, we found that they are entirely different when reactions are induced by the same visual stimuli during reaching movements. The reaction time for a stimulus in the visual periphery was significantly faster than in the central vision, and age-related slowing of reactions to the stimulus in the visual periphery were quite small, compared to that observed in the conventional reaction tasks. This inconsistent slowing of reactions in different motor conditions underscores a distinctive visuomotor pathway for online control, which is more robust against age-related deterioration.

\section{Introduction}

With advancing age, various movements become slower. It is known that this slowing is dominantly composed of two factors: one is a speed decrease (Larsson et al., 1979) due to loss of musculoskeletal functions (Doherty, 2003), and the other is a time prolongation between a stimulus exposure and the onset of a motor response (Der and Deary, 2006), which is primarily caused by the deterioration of sensory and higher neural processing (Schlotterer et al., 1984; Spear, 1993). Reaction time to a visual stimulus is known to be affected not only by aging [longer in elderly adults (Wood, 1999)], but also by its placement in the visual field [longer in the periphery (Osaka, 1976)].

On the other hand, other types of quick reactions to visual stimuli have recently been demonstrated. During reaching movement, the hand can quickly adjust to target displacement (Soechting and Lacquaniti, 1983; Brenner and Smeets, 1997; Day and Lyon, 2000) even without perception of target location change (Goodale et al., 1986; Prablanc and Martin, 1992). Another type of quick and automatic reaction has also been observed when a surrounding visual motion is suddenly given during reaching, called the manual following response (MFR) (Brenner and Smeets, 1997; Whitney et al., 2003; Saijo et al., 2005; Gomi et al., 2006; Gomi, 2008). The reaction latencies of these two types of responses are much faster [100-150 ms vs $>250 \mathrm{~ms}$ for discrimination-reaction tasks (Anzola et al., 1977; Heister et al., 1986)], even though participants in the reaction tasks intently concentrated on detecting the stimulus onset. Therefore, the un-

\footnotetext{
Received June 4, 2009; revised Nov. 6, 2009; accepted Nov. 10, 2009.

We thank S. Shimojo for constructive discussions and continuous support. We also thank P. Haggard and R.C. Miall for helpful discussions and anonymous reviewers for constructive comments and suggestions.

Correspondence should be addressed to Hiroaki Gomi, NTT Communication Science Laboratories, Nippon Telegraph and Telephone Corporation, 3-1 Wakamiya, Morinosato, Atsugi, Kanagawa 243-0198, Japan. E-mail: gomi@idea.brl.ntt.co.jp.

DOI:10.1523/JNEUROSCI.2599-09.2010

Copyright $\odot 2010$ the authors $\quad 0270-6474 / 10 / 300205-05 \$ 15.00 / 0$
}

derlying neural substrates for these online reactions could be distinct from those for the conventional discrimination-reaction tasks, but how visuomotor processes are different for visual field center and periphery and how aging impacts these sensorimotor processes are almost entirely unknown.

We examined whether online responses have different underlying mechanisms from discrimination reactions by comparing how these responses are affected by stimulus location and aging. We measured, in younger and elderly participants, reaction latencies in response to either a central or peripheral visual field stimulus [target jump (TJ), vs background motion (BM), respectively]. Responses were observed either during reaching movements or in a conventional discrimination-reaction task. The results suggest specific neural pathways for online visuomotor controls that are not much affected by age.

\section{Materials and Methods}

Participants. Sixteen younger people (mean age of 28.5 years old, SD of 5.4 years; range of 21-40; 5 females and 11 males, all right handed) and sixteen elderly people (mean age of 68.3 years, SD of 6.0 ; range of $60-78$; 3 females and 13 males, all right handed) participated in this study. None of them reported having any motor or visual disorders. The participants had normal or corrected-to-normal visual acuity. A visual field test before the experiments indicated no neglected field in any of the participants. Written informed consent was obtained from all participants after all experiments had been explained. This experiment was approved by the NTT Communication Science Laboratories Research Ethics Committee.

Experimental setup. All participants took part in two experiments (Fig. 1): one was a discrimination-reaction task with two kinds of visual stimuli, and the other was a visually guided arm reaching task with the same visual stimuli used in the discrimination-reaction task. Participants sat in a darkened room with their heads positioned by a chin rest (height of 23-30 cm; fitted comfortably for each participant). A back-projection screen ( $85 \times 65 \mathrm{~cm}$; RHY-130; Kikuchi Science Laboratory) was placed in front of the participant $40 \mathrm{~cm}$ from the eyes. The visual stimuli were projected with a computer projector (WT610; NEC Viewtechnology).

In the reaching experiment, a wrist splint with an index-finger sleeve was placed on the dominant hand to prevent wrist and index finger 
movements. A reflective marker was placed on the hand dorsum, near the metacarpophalangeal joint of index finger. The marker position was measured with a motion capture system (ProReflex MCU500; Qualisys) at $250 \mathrm{~Hz}$. The axes of coordinates were taken as follows: $x$ was the horizontal direction parallel to the screen, $y$ was the direction perpendicular to the screen, and $z$ was the vertical direction. A button switch connected to the computer to detect the reaching start was placed on the table, $35 \mathrm{~cm}$ from the screen. Two photo diodes (S1223-1; Hamamatsu Photonics) were attached at the bottom corners of screen, and their signals were sent to a computer (sampled at $2 \mathrm{kHz}$ ) for detecting the actual visual stimulus onset.

In the discrimination-reaction experiment, the participant used two buttons (left and right; Fig. 1) placed on the table to indicate the stimulus direction (see below, Visual stimulus and experimental procedure). The reflective markers were attached on the nails of the index and middle fingers to record these movements.

Visual stimulus and experimental procedure. All visual stimuli were generated by a computer (Pentium III, Dual CPU, 1.0 GHz) using Matlab (MathWorks) with Cogent Graphics (University College London, London, UK) software on a Microsoft Windows 2000 operating system. As shown in Figure 1, a white-black random checkerboard pattern [check size of $7 \times 7$ $\mathrm{cm} ; 88.9 \mathrm{~cd} / \mathrm{m}^{2}$ (white); $0.06 \mathrm{~cd} / \mathrm{m}^{2}$ (black); visual angle of $93.5(\mathrm{H})$ by $78.2(\mathrm{~V}) \mathrm{deg}$ ] was presented on a screen at $60 \mathrm{~Hz}$ with $1024 \times 768$ resolution. The center horizontal zone was filled with a gray color $(41.6$ $\mathrm{cd} / \mathrm{m}^{2}, 6.0 \mathrm{~cm}$ height, $8.5 \mathrm{deg}$ visual angle) to mask the checkerboard pattern around the visual field center. At the center of the gray zone, a small filled white circle (diameter $1.0 \mathrm{deg}$ ) was presented as an initial target. The target was shifted rightward or leftward (TJ, $\pm 5 \mathrm{~cm}= \pm 7.1$ $\mathrm{deg}$ ) as the stimulus around the visual field center, and the background checkerboard pattern horizontally moved rightward or leftward (BM, $\pm 190.4 \mathrm{deg} / \mathrm{s}, 1.0 \mathrm{~s}$ ) as the stimulus for the visual periphery.

In the discrimination-reaction experiment, the participants were instructed to place their index and middle fingers on the two buttons (left and right, respectively) while they watched the initial target displayed at the center of the gray zone (Fig. 1). Either the BM or TJ was provided in a single session. The stimulus (BM or TJ) was given in one-third of the trials $(n=30)$ randomly ordered after an interval (1.2-2.1 s, randomly selected) from the trial start beep. The participant was instructed to indicate the direction (left or right) of the stimulus by pushing one of the two buttons as quickly as possible. The participants were also asked not to press the buttons when a stimulus (TJ or BM) was not provided $(n=60)$. All participants took part in the two experimental sessions ( 90 trials/set) for each stimulus condition ( $\mathrm{TJ}$ or $\mathrm{BM}$ ). The order of the conditions was counterbalanced among the participants.

In the reaching experiment, the reaching target was shown at the center of the gray zone at the start of each trial. The participant pressed a button with the index finger to initiate a trial. After a random interval (0.5-1.2 s) from the button-push start, four beeps were presented with a constant interval of $0.6 \mathrm{~s}$. At the third beep, the participant released the button and started reaching. He/she was also instructed to arrive at the target position at around the fourth beep. The participant performed 90 reaching trials. The visual stimulus (BM or TJ) was provided in one-third of the trials ( $n=30$ for each of BM and TJ) $60 \mathrm{~ms}$ after the button release (i.e., reaching start) in random trial order. The participant was also instructed to adjust the reaching movement to the new target as quickly as possible when the target was displaced either rightward or leftward horizontally. Note that the reaching adjustment was not needed in BM trials because the target did not shift position in those trials. This supports the implicit nature of the initial online responses. In the remaining one-third of the trials (control), neither BM nor TJ was applied.

Data analysis. All captured position data were filtered (double-sided, fourth-order Bessel low-pass filter with the cutoff $30 \mathrm{~Hz}$ ), and velocity and acceleration profiles were obtained by three- and five-point numerical time differentiations (without delay), respectively.

The reaction in the discrimination-reaction task was detected by acceleration of finger markers. The reaction time for each participant was obtained from the median value of the intervals between stimulus onset and the finger movement onset of all successful trials. Here, finger movement onset was detected by the threshold for the $z$-directional (vertical) acceleration of the finger markers, which was defined as seven times the $\mathrm{SD}$ of the acceleration during the period of $100 \mathrm{~ms}$ just before the visual stimulus onset. Note that there was no significant difference $\left(t\right.$ test, $t_{(30)}=$ 1.627, $p=0.1142$ ) in the correct-trial rate (the button was correctly pressed to indicate the stimulus direction) for TJ between the elderly $(99.0 \pm 1.2 \%)$ and younger $(99.6 \pm 1.0 \%)$ groups, but the correct-trial rate for BM was significantly lower $\left(t\right.$ test, $t_{(30)}=2.797, p=0.0089$ ) for the elderly group $(93.5 \pm 6.1 \%)$ than for the younger group (98.2 \pm $2.7 \%$ ). The trial ratio of no-response (defined as no reaction during the interval of $1.0 \mathrm{~s}$ after stimulus onset) for BM was significantly higher $\left(t\right.$ test, $\left.t_{(30)}=2.550, p=0.0161\right)$ in the elderly group $(4.8 \pm 4.2 \%)$ than in the younger group $(1.7 \pm 2.6 \%)$, suggesting reduced peripheral visual sensitivity in the elderly group in this task.

For the reaching data, to exclude outliers that deviated from the other trials in each stimulus condition, we chose 24 of 30 trials by means of the least root mean square of the trajectory deviation from the median trajectory. Note that trials in which participants made obvious reaching errors (e.g., long start delay, mistaken adjustment) were excluded (max: two trials in one stimulus condition) from the calculation of the median trajectory. Regarding the reaching duration, a two-way (age-group $\times$ stimulus condition) mixed-model ANOVA with repeated measures did not show any significant difference in the main effect of age group $\left(F_{(1,30)}=\right.$ $2.145, p=0.1535)$ and in the interaction between the two factors $\left(F_{(2,28)}=\right.$ 

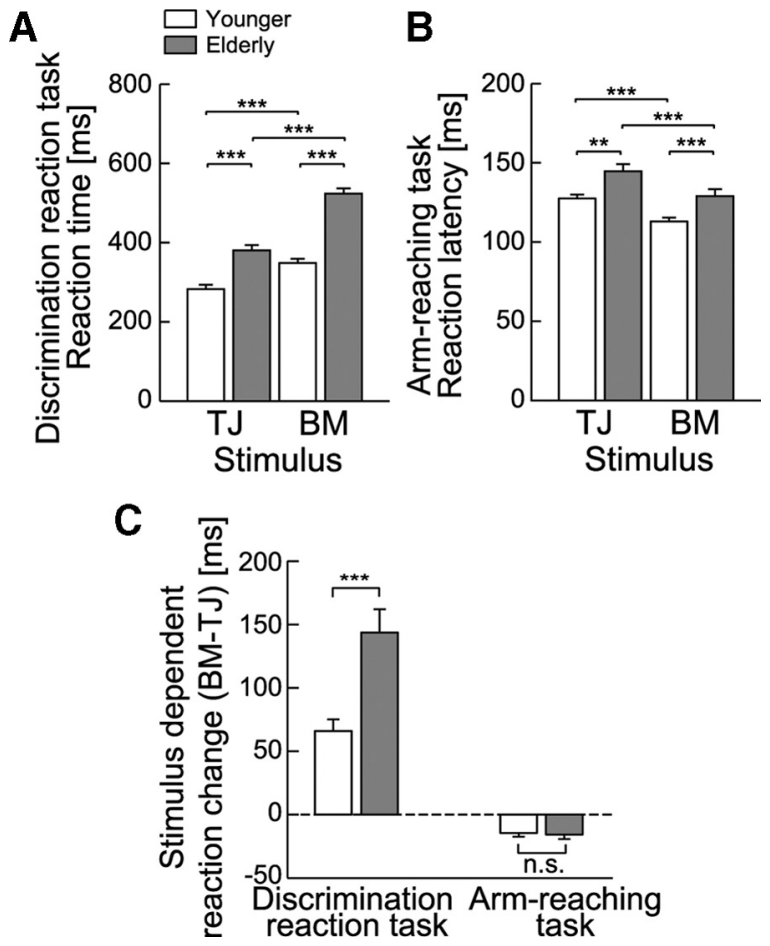

Figure 2. Impact of aging on reactions to visual stimulus directions. A, Reaction times for TJ in the visual field center and $B M$ in peripheral vision in the discrimination-reaction tasks for the younger (open bar) and elderly (gray bar) groups. $\boldsymbol{B}$, Reaction latencies for TJ and BM in the reaching tasks for the two participant groups. C, Stimulus-dependent reaction change (BM TJ) for the two participant groups (open bar: younger, gray bar: elderly) in each task. In all graphs, asterisks $\left(^{* *}\right.$ and $\left.{ }^{* * *}\right)$ denote the significance of differences: $p<0.01$ and $p<0.001$, respectively. The error bar denotes SEM.

$0.245, p=0.7846)$. The main effect of stimulus condition was significant $\left(F_{(2,60)}=4.867, p=0.0110\right)$.

To characterize the responses for TJ and BM, we focused on the difference between the mean $x$-acceleration profiles for the leftward and rightward stimuli in each stimulus condition (TJ or BM). Reaction latencies to the TJ and BM for each participant were defined as the time from which the significant difference by the successive $t$ test $(p<0.05)$ between the $x$-directional acceleration responses for the rightward and leftward stimuli continued for at least $20 \mathrm{~ms}$ in a window between 0 and $300 \mathrm{~ms}$.

\section{Results}

Figure 2 compares the age-related impact on the reaction times for the central (TJ) and peripheral visual stimuli (BM) given in the conventional discrimination-reaction tasks (Fig. 2A) and in the reaching tasks (Fig. $2 B$ ). Reaction time was analyzed in a mixed model ANOVA with age group as the between-subject factor and stimulus condition as the within-subject factor. We found significant effects of age group $\left(F_{(1,30)}=48.989, p<\right.$ $0.00001)$, stimulus $\left(F_{(1,30)}=103.312, p<0.00001\right)$, and their interaction $\left(F_{(1,30)}=14.167, p=0.00073\right)$. The reaction times for the $\mathrm{TJ}$ and $\mathrm{BM}$ stimuli were $331.5 \pm 12.1 \mathrm{~ms}$ and $436.3 \pm 20.2 \mathrm{~ms}$ across all participants, respectively. A post hoc t test indicated that, in the younger group, the reaction time detected by finger acceleration for BM (mean \pm SEM $348.9 \pm 14.6 \mathrm{~ms}$ ) was significantly longer (paired $t$ test, $t_{(15)}=7.252, p=<0.00001$ ) than that for TJ (282.9 $\pm 10.6 \mathrm{~ms}$ ) (Fig. $2 \mathrm{~A}$ ). This reaction-time prolongation for the peripheral stimuli (BM) was also significant (paired $t$ test, $t_{(15)}=$ $7.761, p<0.00001$ ) in the elderly group, and it was considerably greater $\left(77.6 \mathrm{~ms}\right.$ in mean; $t$ test, $\left.t_{(30)}=3.764, p=0.00007\right)$ than that in the younger group (Fig. $2 C$, compare left two bars).
Hence, this result is consistent with the general view that the processing for the peripheral vision deteriorates more with aging (Crassini et al., 1988; Wood, 1999).

If these reaction time differences between central and peripheral stimuli and their prolongations with aging are simply due to the inhomogeneous characteristics of visual processing (Johnston and Wright, 1985; Crassini et al., 1988) or difference between visual stimuli, similar reaction latency changes should be observed when identical stimuli are given during reaching. However, our findings did not confirm this prediction. Figure $3 A$ shows a younger participant's typical mean trajectories projected on the horizontal plane for TJ and BM (rightward and leftward of both stimuli). In the TJ conditions (dashed curves), the hand trajectories started to deviate after a short delay from the stimulus onsets (indicated by a triangle) toward the new target location (left or right). These corrective responses were clearly observed in hand $x$-acceleration temporal profiles (Fig. $3 B$, dashed curves) for both younger (top) and elderly (bottom) participants. The initial parts of these responses are generated by an implicit (or involuntary) visuomotor process, which is thought to assist the quick reaching correction (Goodale et al., 1986; Day and Lyon, 2000). In the BM condition (Fig. $3 A, B$, solid curves), on the other hand, the hand trajectories deviated from the control as well. These deviations are also regarded as implicit visuomotor reactions (MFR) (Saijo et al., 2005; Gomi et al., 2006; Gomi, 2008). As shown in Figure $3 A$, these deviations of trajectories were readjusted in the later phase of reaching so that the reaching went to the center visible target.

Next, reaction latencies were determined as the onset of acceleration deviation. Open and filled triangles in Figure $3 B$ indicate the statistically detected latencies of manual responses for TJ and BM, respectively. Unlike the long prolongation in the discriminationreaction time with aging shown in Figure $2 A$, the detected reaction latencies for TJ and BM of this particular elderly participant (Fig. 3B, bottom) in the reaching task were not much delayed from those of the younger participant (Fig. 3B, top).

As shown in the group analysis with mixed model ANOVA (Fig. 2B), the reaction latencies both for the central (TJ) and peripheral (BM) stimuli during reaching were not much affected by aging: there was a significant $\left(F_{(1,30)}=17.876, p=0.0002\right)$ but small effect of aging. Group mean latency differences were only $17.2 \mathrm{~ms}$ and $16.0 \mathrm{~ms}$ in mean for TJ and BM, respectively. The other main factor, visual stimulus, was also significant $\left(F_{(1,30)}=\right.$ $43.340, p<0.00001,136.1 \pm 2.9 \mathrm{~ms}$ for TJ and $121.0 \pm 2.5 \mathrm{~ms}$ for $\mathrm{BM}$ across all participants), but the interaction between age group and stimulus was not significant $\left(F_{(1,30)}=0.074, p<\right.$ 0.7875), unlike in the discrimination-reaction task. Post hoc comparisons indicate that the reaction latencies for TJ were slightly but significantly longer than the reaction latencies for BM, both for the younger (paired $t$ test, $t_{(15)}=4.930, p=0.00002$ ) and elderly groups (paired $t$ test, $t_{(15)}=4.462, p=0.00005$ ) as shown in Figure $2 B$. This tendency was opposite that in the discriminationreaction tasks (the right two bars are negative whereas left two bars are positive in Fig. 2C). Further, the most striking discrepancy is that the difference between reaction latencies for TJ and BM stimuli during reaching in the elderly group was not significantly different $\left(t\right.$ test, $t_{(30)}=0.272, p=0.7875$ ) from that in the younger group (Fig. 2C, compare right two bars), whereas that latency difference in the discrimination-reaction task was markedly prolonged by aging (Fig. 2C, compare left two bars). Note that, under the observed variance of the latency difference $(\mathrm{SD}=12.8 \mathrm{~ms})$ and the sample size of each group $(n=16)$ in the reaching task, the smallest distinguishable difference was $13.1 \mathrm{~ms}$ obtained by statistical power analysis 
(effect size $=0.8$ ), which was markedly smaller than the mean difference in the discrimination-reaction task.

\section{Discussion}

These remarkable discrepancies in reaction behavior in different motor task conditions indicate that visuomotor processing in the reaching tasks is different from that in the discrimination-reaction tasks: the former is not sensitive to stimulus position, and is only minimally affected by aging. Indeed, the reactions to the identical stimuli applied during reaching were much quicker than those in the discrimination-reaction tasks even though those reactions were similarly detected by initiations of stimulus-driven changes of movement acceleration. One could speculate that the methodological difference in reaction detection for these tasks might produce the change in the trends in reaction time. However, all trends in reaction time variations with aging and with different stimuli in the discriminationreaction task were the same (i.e., two main effects and interaction were significant, $p<0.05$, in the mixed model ANOVA) even when the reaction time was detected by the successive $t$ test with large outlier trimming (33\% percentile cutoff) for the finger velocity profiles.

What aspect of visuomotor processing is different between these two task conditions? Previous studies have shown that the aging-related retardation (30-40 ms) of visually evoked potential, which is related to the early stage of visual processing (Fiorentini et al., 1996), is comparable to the age-related slowing of simple reaction to a visual motion onset (Porciatti et al., 1999). This suggests that the prolongation of reaction time with aging is largely due to the sensory factors, even though it may be partly due to the motor factors. More strikingly, the difference between reaction times to $\mathrm{BM}$ and $\mathrm{TJ}$ in the discrimination-reaction task was significantly longer for the elderly group than for the younger group. This cannot be explained by the effect of age-dependent slowing of movement initiation (i.e., motor factor), which was subtracted in each age group. For a choice task, on the other hand, reaction time increases with the number of choices available and is greatly prolonged with aging (Yordanova et al., 2004; Der and Deary, 2006), whereas reaction time in a simple reaction task is rather insusceptible to aging (Porciatti et al., 1999; Yordanova et al., 2004). Considering the fact that the age-related increases of reaction latencies during reaching were small, the great increases of reaction times with aging in our discrimination-reaction tasks (reaction time increase with aging for TJ: mean $97.3 \mathrm{~ms}, t$ test, $t_{(30)}=5.669, p=0.00001$; for BM: mean $174.9 \mathrm{~ms} t$ test, $t_{(30)}=$ $6.735, p=0.00001$ ) are not simply due to the deterioration of the early stage of visual processing. Instead, these reaction-time increases appear to be dominantly due to the prolongation of processing for discrimination and action selection.

An additional intriguing aspect of our results is that the reaction latency for BM given during reaching did not much change with aging, but the great age-related prolongation of reaction time for the identical stimulus was observed in the discrimination-
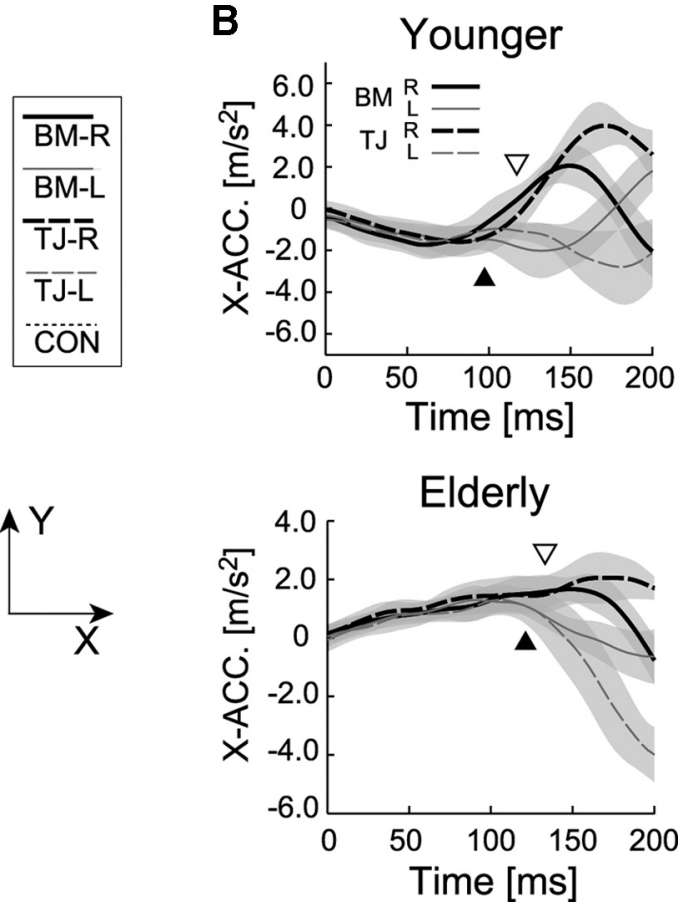

$\overline{\mathrm{BM}-\mathrm{R}}$ $\overline{B M-L}$ $\overline{\mathrm{TJ}} \overline{\mathrm{R}}$ $\overline{\mathrm{T} J}-\overline{\mathrm{L}}$ COON Time $[\mathrm{ms}]$ for the TJ and BM stimuli (R, rightward, L, leftward) of a younger participant (top) and an elderly (bottom) participant. Open and filled triangles denote the reaction latencies detected statistically for TJ and BM stimulus conditions, respectively.

reaction task. This peripheral-vision-specific slowing with aging in the discrimination-reaction task further indicates that the processing of discrimination itself, rather than early visual processing, for the peripheral visual input is susceptible to aging.

Why, on the other hand, were the reaction latencies of the responses during reaching not much influenced by aging? After all, similar computation of stimulus discrimination and action selection are also necessary to produce the stimulus-directiondependent reaction (right or left) during reaching. The key to understanding these phenomena lies in the underlying mechanisms of these computations in the online controls. For the sake of quick reaction, implicit sensorimotor processes appear to use continuous and direct sensorimotor transformations rather than the action selection or switching mechanism usually assumed in the classical reaction model (Donders, 1969; Ghez and Krakauer, 2000; Miller and Low, 2001).

For responses to target displacement, it has been suggested that the posterior parietal cortex is involved in the continuous error correction, in which a predictive forward model of the hand movement is computed (Desmurget and Grafton, 2000). For peripheral visual motion, extremely fast visual motion analysis, which is less affected ( $<40 \mathrm{~ms}$ ) by aging (Porciatti et al., 1999), may continuously send motion information to motor coordination process via a specific neural pathway, which has been partly investigated in recent studies (Gomi, 2008). The reversal of the reaction time lengths for central and peripheral stimuli by the motor task change (Fig. 2C) supports the idea of distinct sensorimotor transformation for the surrounding visual motion.

Together, the results suggest that different types of visuomotor processing are selected according to the environmental (stimulusrelated) and motor situation. This is consistent with the idea of multiple visual processing streams (Ungerleider and Haxby, 1994; 
Pettersson et al., 1997; Desmurget et al., 1999; Rossetti et al., 2003; Prado et al., 2005), especially for perception and action (Goodale and Milner, 1992), and suggests that these processing streams may be differentially affected by aging.

\section{References}

Anzola GP, Bertoloni G, Buchtel HA, Rizzolatti G (1977) Spatial compatibility and anatomical factors in simple and choice reaction time. Neuropsychologia 15:295-302.

Brenner E, Smeets JB (1997) Fast responses of the human hand to changes in target position. J Mot Behav 29:297-310.

Crassini B, Brown B, Bowman K (1988) Age-related changes in contrast sensitivity in central and peripheral retina. Perception 17:315-332.

Day BL, Lyon IN (2000) Voluntary modification of automatic arm movements evoked by motion of a visual target. Exp Brain Res 130:159-168.

Der G, Deary IJ (2006) Age and sex differences in reaction time in adulthood: results from the United Kingdom Health and Lifestyle Survey. Psychol Aging 21:62-73.

Desmurget M, Grafton S (2000) Forward modeling allows feedback control for fast reaching movements. Trends Cogn Sci 4:423-431.

Desmurget M, Epstein CM, Turner RS, Prablanc C, Alexander GE, Grafton ST (1999) Role of the posterior parietal cortex in updating reaching movements to a visual target. Nat Neurosci 2:563-567.

Doherty TJ (2003) Invited review: aging and sarcopenia. J Appl Physiol 95:1717-1727.

Donders FC (1969) On the speed of mental processes. Acta Psychol (Amst) 30:412-431.

Fiorentini A, Porciatti V, Morrone MC, Burr DC (1996) Visual ageing: unspecific decline of the responses to luminance and colour. Vision Res 36:3557-3566.

Ghez C, Krakauer J (2000) The organization of movement. In: Principles of neural science, Ed 4 (Kandel ER, Schwartz JH, Jessell TM, eds), pp 653 673. New York: McGraw-Hill.

Gomi H (2008) Implicit online corrections of reaching movements. Curr Opin Neurobiol 18:558-564.

Gomi H, Abekawa N, Nishida S (2006) Spatiotemporal tuning of rapid interactions between visual-motion analysis and reaching movement. J Neurosci 26:5301-5308.

Goodale MA, Milner AD (1992) Separate visual pathways for perception and action. Trends Neurosci 15:20-25.

Goodale MA, Pelisson D, Prablanc C (1986) Large adjustments in visually guided reaching do not depend on vision of the hand or perception of target displacement. Nature 320:748-750.

Heister G, Ehrenstein WH, Schroeder-Heister P (1986) Spatial S-R compatibility effects with unimanual two-finger choice reactions for prone and supine hand positions. Percept Psychophys 40:271-278.
Johnston A, Wright MJ (1985) Lower thresholds of motion for gratings as a function of eccentricity and contrast. Vision Res 25:179-185.

Larsson L, Grimby G, Karlsson J (1979) Muscle strength and speed of movement in relation to age and muscle morphology. J Appl Physiol 46:451-456.

Miller JO, Low K (2001) Motor processes in simple, go/no-go, and choice reaction time tasks: a psychophysiological analysis. J Exp Psychol Hum Percept Perform 27:266-289.

Osaka N (1976) Reaction time as a function of peripheral retinal locus around fovea: effect of stimulus size. Percept Mot Skills 42:603-606.

Pettersson LG, Lundberg A, Alstermark B, Isa T, Tantisira B (1997) Effect of spinal cord lesions on forelimb target-reaching and on visually guided switching of target-reaching in the cat. Neurosci Res 29:241-256.

Porciatti V, Fiorentini A, Morrone MC, Burr DC (1999) The effects of ageing on reaction times to motion onset. Vision Res 39:2157-2164.

Prablanc C, Martin O (1992) Automatic control during hand reaching at undetected two-dimensional target displacements. J Neurophysiol 67: 455-469.

Prado J, Clavagnier S, Otzenberger H, Scheiber C, Kennedy H, Perenin MT (2005) Two cortical systems for reaching in central and peripheral vision. Neuron 48:849-858.

Rossetti Y, Pisella L, Vighetto A (2003) Optic ataxia revisited: visually guided action versus immediate visuomotor control. Exp Brain Res 153:171-179.

Saijo N, Murakami I, Nishida S, Gomi H (2005) Large-field visual motion directly induces an involuntary rapid manual following response. J Neurosci 25:4941-4951.

Schlotterer G, Moscovitch M, Crapper-McLachlan D (1984) Visual processing deficits as assessed by spatial frequency contrast sensitivity and backward masking in normal ageing and Alzheimer's disease. Brain 107:309-325.

Soechting JF, Lacquaniti F (1983) Modification of trajectory of a pointing movement in response to a change in target location. J Neurophysiol 49:548-564.

Spear PD (1993) Neural bases of visual deficits during aging. Vision Res 33:2589-2609.

Ungerleider LG, Haxby JV (1994) 'What' and 'where' in the human brain. Curr Opin Neurobiol 4:157-165.

Whitney D, Westwood DA, Goodale MA (2003) The influence of visual motion on fast reaching movements to a stationary object. Nature 423:869-873.

Wood JM (1999) How do visual status and age impact on driving performance as measured on a closed circuit driving track? Ophthalmic Physiol Opt 19:34-40.

Yordanova J, Kolev V, Hohnsbein J, Falkenstein M (2004) Sensorimotor slowing with ageing is mediated by a functional dysregulation of motorgeneration processes: evidence from high-resolution event-related potentials. Brain 127:351-362. 